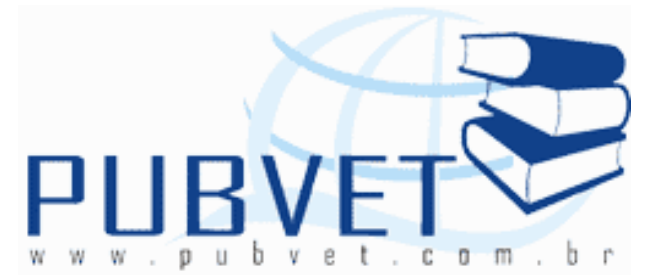

PUBVET, Publicações em Medicina Veterinária e Zootecnia.

\title{
Termorregulação de bovinos em ambiente tropical: uma abordagem com ênfase nas respostas fisiológicas
}

\section{Maiko Roberto Tavares Dantas ${ }^{1}$, João Batista Freire de Souza Junior ${ }^{1}$, Hérica Girlane Tertulino Domingos ${ }^{1}$, Janio Lopes Torquato ${ }^{1}$, Geovan Figueirêdo Sá Filho $^{1}$, Leonardo Lelis de Macedo Costa ${ }^{1}$}

${ }^{1}$ Universidade Federal Rural do Semi-Árido, Departamento de Ciências Animais, Laboratório de Biometeorologia, Biofísica Ambiental e Bem-Estar Animal (LABBEA), BR 110, Km 47, Costa e Silva, CEP: 59625-900, Mossoró-RN.

\section{Resumo}

O efeito do ambiente térmico sobre as respostas fisiológicas de bovinos leiteiros tem sido bastante estudado como uma forma de caracterizar situações de estresse térmico causado por tensões exercidas pelo ambiente o qual os animais estão inseridos. As exigências térmicas dos animais variam de acordo com o estágio de desenvolvimento sendo que, na fase de aleitamento, os bezerros possuem uma capacidade termorregulatória pouco desenvolvida e, o planejamento de instalações é fundamental, pois facilitam o manejo e protegem os animais de condições meteorológicas adversas. Assim, objetivouse com esta revisão abordar os principais aspectos da termorregulação de bovinos em ambiente tropical com ênfase na frequência respiratória, temperatura retal e da superfície corporal. A temperatura superficial, por estar diretamente exposta ao ambiente, apresenta variações consideráveis, as quais 
acompanham as variações dos componentes meteorológicos do ambiente em que estão inseridos. Já a temperatura retal é uma resposta tardia ao estresse térmico, com pouca ou nenhuma variação. Ao contrário da temperatura retal, a frequência respiratória é o primeiro sinal visível que evidencia uma situação de estresse calórico. Assim, para evitar situações térmicas que impeçam os animais de demonstrarem todo o seu potencial produtivo, além de evitar danos na saúde e reprodução, deve-se fornecer um microclima caracterizado por baixas temperaturas e sem a exposição à radiação solar direta.

Palavras-chave: estresse térmico, frequência respiratória, temperatura retal, temperatura superficial.

\title{
Thermoregulation of cattle in tropical environment: an approach with emphasis on physiological responses
}

\begin{abstract}
The effect of thermal environment on the physiological responses of dairy cattle has been extensively studied as a way of characterizing situations of thermal stress caused by tension exerted by the environment to which animals are placed. The thermal requirements of animals vary with the stage of development is that, during the breast-feeding, the calves have a poorly developed thermoregulatory capacity and facilities planning is essential, because they facilitate the management and protecting the animals from adverse weather conditions. Therefore, aimed of this review address the main aspects of thermoregulation in cattle in tropical environment with emphasis on respiratory rate, rectal temperature and body surface. The surface temperature, because it is directly exposed to the environment, varies considerably, which accompany the variations of the meteorological components of the environment in which they live. Since the rectal temperature is a late response to heat stress, with little or no variation. Unlike the rectal temperature, respiratory rate is the first visible sign that shows a heat stress situation. So, to avoid thermal situations that prevent animals to
\end{abstract}


DANTAS, M.R.T. et al. Termorregulação de bovinos em ambiente tropical: uma abordagem com ênfase nas respostas fisiológicas. PUBVET, Londrina, V. 6, N. 7, Ed. 194, Art. 1306, 2012.

demonstrate all their potential, and avoid damage to the health and reproduction, must provide a microclimate characterized by low temperatures and without exposure to direct sunlight.

Keywords: heat stress, respiratory rate, rectal temperature, surface temperature.

\section{INTRODUÇÃO}

Condições térmicas adversas ocorrem no ambiente natural dos animais, causando respostas ao estresse, levando a uma redução no desempenho produtivo e reprodutivo. Dentro de certos limites, os animais podem se ajustar fisiológica, comportamental ou imunologicamente de modo a sustentar a homeostase orgânica e assim minimizar as conseqüências adversas. Entretanto, neste processo de ajuste podem ser atingidas funções menos vitais ao organismo, como o desempenho (produção, reprodução, eficiência) ou bem-estar, quando a intensidade e a duração dos estressores ambientais excedem a capacidade compensatória do organismo, a qual é geneticamente determinada.

Em regiões de baixa latitude, a temperatura do ar tende a ser muito próxima ou até superior àquela da superfície corporal, tornando a convecção ineficaz; em adição, se o ambiente for caracterizado por intensa radiação solar, o animal passa a ganhar calor por radiação (SILVA, 2000a). Obviamente, a habilidade de um animal resistir a esse ambiente é proporcional à sua capacidade em dissipar calor latente na superfície corporal como resultado da sudação (FINCH et al., 1982; McLEAN, 1963b), no sistema respiratório (STEVENS, 1981; SILVA et al., 2002), ou ainda na capacidade dos animais estocarem calor em seus tecidos (FINCH, 1985; McLEAN et al., 1963ab), resultando na elevação da temperatura corporal.

Segundo Ferreira et al. (2006) a susceptibilidade dos bovinos ao estresse calórico aumenta à medida que o binômio umidade relativa e temperatura ambiente ultrapassa a zona de conforto térmico, o que dificulta a dissipação de 
DANTAS, M.R.T. et al. Termorregulação de bovinos em ambiente tropical: uma abordagem com ênfase nas respostas fisiológicas. PUBVET, Londrina, V. 6, N. 7, Ed. 194, Art. 1306, 2012.

calor que, por sua vez, aumenta a temperatura corporal, com efeito negativo sobre o desempenho. Segundo o mesmo autor, o estresse calórico promove alterações na homeostase e tem sido quantificado mediante mensuração de variáveis fisiológicas tais como, temperatura retal, freqüência respiratória e concentrações hormonais, onde a capacidade do animal de resistir aos rigores deste estresse, tem sido avaliada fisiologicamente por alterações na temperatura retal e na frequência respiratória.

Neste contexto, objetivou-se com esta revisão abordar os principais aspectos da termorregulação de bovinos em ambiente tropical com ênfase na frequência respiratória, temperatura retal e da superfície corporal.

\section{TERMORREGULAÇÃO}

Ambiente é o conjunto de tudo o que interfere e afeta a constituição, o comportamento e a evolução de um organismo e que não envolve diretamente fatores genéticos (SILVA, 2000a). Portanto, um animal e seu ambiente formam um sistema em que ambos agem um sobre o outro. As regiões tropicais se caracterizam por elevados níveis de radiação solar e temperatura ambiente, fatores que afetam adversamente a produção animal, quando comparada à de animais mantidos em zonas temperadas (JOHNSON, 1987). Segundo Curtis (1983), citado por Baeta \& Souza (1997), o intervalo de temperatura da zona de conforto térmico para bovinos recém-nascidos está compreendido entre $18^{\circ} \mathrm{C}$ a $21^{\circ} \mathrm{C}$ (região ótima), sendo que a temperatura efetiva crítica inferior (TCI) é de $10^{\circ} \mathrm{C}$ e a temperatura efetiva crítica superior (TCS) de $26^{\circ} \mathrm{C}$. Os efeitos da temperatura e da umidade do ar são, geralmente, os principais fatores limitantes para o desenvolvimento, produção e reprodução dos animais, em razão do estresse causado (NÄÄS et al., 2001).Sob condições naturais, o ambiente térmico é bastante complexo porque a radiação, a velocidade do vento, a temperatura e a umidade do ar modificam-se no tempo e no espaço. 
DANTAS, M.R.T. et al. Termorregulação de bovinos em ambiente tropical: uma abordagem com ênfase nas respostas fisiológicas. PUBVET, Londrina, V. 6, N. 7, Ed. 194, Art. 1306, 2012.

Estes fatores interagem entre si e com as diferentes características do organismo, em conseqüência de que a alteração de qualquer variável ambiental pode provocar variações comportamentais e fisiológicas dos organismos em resposta à necessidade de manutenção do equilíbrio térmico homeotermia - nos animais, configurando a condição de estresse (FINCH, 1984). Sendo assim, a habilidade para regular a temperatura é uma adaptação evolucionária que permitiu aos animais homeotérmicos sustentarem as suas funções, apesar das variações térmicas do ambiente (BAKER, 1989).

A homeotermia pode ser expressa pela equação de balanço térmico: Produção de calor $=$ perda de calor \pm estoque de calor. Quando um mamífero produz calor excessivo pelo metabolismo ou ganha do ambiente, estabelece-se a hipertemia. Ao contrário, se ocorre perda excessiva de calor, torna-se hipotérmico (YOUSEF,1987).

O processo de controle da temperatura em um sistema biológico qualquer é chamado de Termorregulação. $O$ balanço de energia térmica presente em um animal homeotérmico, tal como um bovino, pode ser expresso pela seguinte equação:

$$
M+R_{C} \pm R_{L} \pm C_{R} \pm C_{S} \pm K-E_{S}-E_{R}=0
$$

onde $M\left(\mathrm{~W} \mathrm{~m}^{-2}\right)$ é a taxa de produção de calor liberada nos processos metabólicos, ou seja na oxidação dos alimentos ou das reservas corporais. $R_{C}$ $\left(\mathrm{W} \mathrm{\textrm {m } ^ { - 2 }}\right.$ ) é o ganho de calor através da radiação de ondas curtas, $R_{L}, K, C_{R}$ e $C_{S}$ $\left(\mathrm{W} \mathrm{m}^{-2}\right)$ são as trocas de energia térmica por radiação de ondas longas e os fluxos de calor por condução, por convecção respiratória e por convecção na superfície do pelame, $E_{S}$ e $E_{R}\left(\mathrm{~W} \mathrm{~m}^{-2}\right)$ são as perdas de calor latente através da evaporação cutânea e respiratória.

Os termos $R_{C}, L, C$ e $K$ representam a perda de calor sensível (não evaporativo), enquanto $E_{R}$ e $E_{C}$ representam o calor latente (evaporativo). Em altas latitudes, a temperatura ambiente encontra-se geralmente abaixo da 
DANTAS, M.R.T. et al. Termorregulação de bovinos em ambiente tropical: uma abordagem com ênfase nas respostas fisiológicas. PUBVET, Londrina, V. 6, N. 7, Ed. 194, Art. 1306, 2012.

temperatura crítica inferior. Neste caso, predomina a perda de calor sensível para o ambiente, enquanto a temperatura corporal do animal se mantém estável (MCLEAN, 1963a), conforme mostra a Figura 1. Em contraste, nas baixas latitudes a temperatura ambiente com freqüência excede a temperatura crítica superior, passando a predominar a dissipação de calor latente, enquanto o metabolismo declina (TANEJA, 1958 e 1959; McLEAN, 1963ab; FINCH, 1985; ARKIN et al., 1991; SILVA et al., 2002).

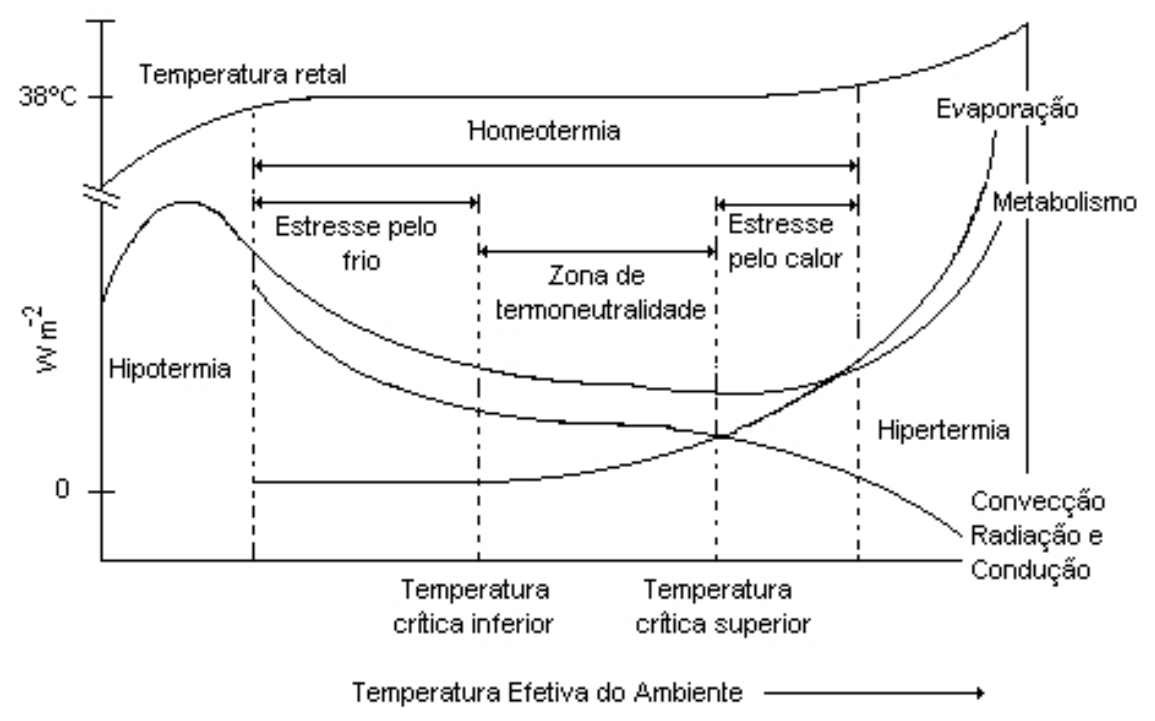

Figura 1 Respostas básicas da termorregulação em relação à temperatura efetiva do ambiente. Adaptado de Esmay (1969), Ehrlemark e Sallvik (1996).

A resposta ao estresse térmico compreende o reconhecimento da ameaça à homeostase ou ao bem-estar do organismo, a resposta de estresse e as conseqüências do estresse (MORBERG, 1976). São vários os fatores que controlam a natureza da resposta biológica de um animal a um estressor, esses podem ser a genética, a idade, experiência anterior, sexo ou condições fisiológicas. Os mecanismos de controle térmico podem ser de três tipos: comportamentais, autônomos e adaptativos, os quais são integrados em centros termorreguladores do hipotálamo (SILVA, 2000a). A resposta homeostática geral ao estresse térmico em mamíferos inclui vasodilatação 
DANTAS, M.R.T. et al. Termorregulação de bovinos em ambiente tropical: uma abordagem com ênfase nas respostas fisiológicas. PUBVET, Londrina, V. 6, N. 7, Ed. 194, Art. 1306, 2012.

periférica, aumento da freqüência respiratória, alta taxa de sudação, maior ingestão de água e menor de alimentos, redução do metabolismo, o que resulta em menor produção de leite, menor ganho de peso e queda na fertilidade do rebanho.

Os sistemas de termorregulação dos animais garantem sua sobrevivência e outras funções metabólicas (crescimento, lactação, reprodução, etc.). Na atualidade, não se tem mais dúvida de que a necessidade de manter a homeotermia, ou os processos usados pelo organismo para mantê-la, é que afetam negativamente o desempenho animal em condições adversas de temperatura (JORDAN, 2003; GARCIA-ISPIERTO, et al., 2005)

A região semi-árida é caracterizada por altas temperaturas e elevados níveis de radiação que afetam diretamente o equilíbrio térmico dos animais de interesse zootécnico, e consequentemente, seu potencial produtivo. A manutenção da homeotermia é prioridade para os animais e impera sobre as funções produtivas como produção de leite e reprodução. No Semi-Árido as temperaturas ambientes são, na maior parte do dia, elevadas, estando acima da zona de termoneutralidade (MARTELLO et al., 2004).

Nestas condições, os mecanismos sensíveis de transferência térmica, ou seja, radiação, convecção e condução podem tornar-se mecanismos de ganho de calor, pois dependem diretamente do gradiente de temperatura entre o animal e ambiente (McLEAN, 1963b; GEBREMEDHIN et al., 2001; SILVA, 2000; MAIA et al., 2005a). Por outro lado, a evaporação torna-se a principal via para a dissipação de energia térmica dos animais (FINCH, 1985; GEBREMWDHIN et al., 1981), pois não depende desse gradiente de temperatura, a qual ocorre na superfície da epiderme, pela sudação (McLEAN, 1963b; TANEJA, 1958 e 1959; SILVA e STALING, 2003; MAIA et al., 2005a), e no trato respiratório (STEVENS, 1981; SILVA et al., 2002; MAIA et al., 2005b). 


\section{RESPOSTAS FISIOLÓGICAS}

Os efeitos do ambiente térmico sobre as respostas fisiológicas de bovinos leiteiros como a freqüência respiratória, temperatura retal e temperatura da superfície do pelame têm sido bastante estudados como uma forma de caracterizar situações de estresse térmico causado por tensões exercidas pelo ambiente o qual os animais estão inseridos.

\subsection{FREQUÊNCIA RESPIRATÓRIA}

A freqüência respiratória está sujeita a variações intrínsecas e extrínsecas. As intrínsecas caracterizam-se pelas respostas aos exercícios físicos, medo, excitação, estado fisiológico e produção de leite (Wolff e Monty, 1974; Stöber, 1993; Carvalho et al., 1995; Marai et al., 1999). Fatores extrínsecos são atribuídos ao ambiente, como condições climáticas, principalmente temperatura e umidade do ar, radiação solar, velocidade dos ventos, estação do ano, hora do dia, densidade e sombreamento (Ingraham et al., 1979; Igono et al., 1985; Okantah et al., 1992; Muller e Botha, 1993; Muller et al., 1994a; Pires et al., 1998b; Marai et al., 1999). A freqüência respiratória normal em bovinos adultos varia entre 24 e 36 movimentos respiratórios por minuto ( $\mathrm{mov} / \mathrm{min}$ ) (Stöber, 1993), mas pode apresentar valores mais amplos, entre 12 e 36 mov/min (Terra, 1993). Sob estresse térmico, a freqüência respiratória começa a elevar-se antes da temperatura retal (Bianca, 1965) e, geralmente, observa-se taquipnéia em bovinos em ambientes com temperatura elevada (Stöber, 1993; Muller et al., 1994b; Pires et al., 1998a).

Alterações na freqüência respiratória são usadas por diversas espécies animais para perder calor para o ambiente pela via respiratória; o seu aumento tem sido descrito por vários autores (BORUT et al., 1979; DMI'EL e ROBERTSHAW, 1983; GAYÃO, 1992; SANTOS, 2003) como sendo a primeira resposta corporal à elevação da temperatura do ar. No ambiente tropical, o 
DANTAS, M.R.T. et al. Termorregulação de bovinos em ambiente tropical: uma abordagem com ênfase nas respostas fisiológicas. PUBVET, Londrina, V. 6, N. 7, Ed. 194, Art. 1306, 2012.

fator que mais afeta os animais é o aporte térmico devido à radiação solar intensa, tanto em forma direta como indireta (re-irradiação da energia térmica pelas superfícies ambientes circunvizinhas).

Nos ungulados a ofegação é uma importante via de perda de calor, o que tem sido explicado por certos autores como uma forma de resfriamento seletivo do cérebro que auxilia na manutenção da temperatura cerebral abaixo da temperatura média do corpo (JESSEN; PONGRATZ, 1979). Entretanto, o aumento do trabalho respiratório é na realidade uma desvantagem devido ao considerável calor gerado pelos músculos respiratórios, o qual é maior que o que pode ser dissipado, atenuado pela propriedade elástica do sistema respiratório (SCHMIDT-NIELSEN, 2002). Uma reação do organismo ao estresse é o aumento da secreção de adrenalina e noradrenalina pelas glândulas adrenais para a circulação sanguínea. Elevadas concentrações destes dois hormônios na corrente sanguínea resulta na aceleração dos batimentos cardíacos.

O incremento da temperatura interna, caracterizado pela temperatura retal induz a necessidade do animal dissipar esse excedente de energia, levando ao aumento da freqüência respiratória. Sob condições controladas de temperatura e umidade, Jessen e Pongratz (1979) observaram que a freqüência respiratória declinava imediatamente quando a umidade do ar passou de 37 a 95,7\%. O aumento observado pode ser explicado como uma tentativa desses animais em perder calor pela via respiratória principalmente pelo mecanismo convectivo, uma vez que houve diminuição pela perda evaporativa respiratória, inibida pela alta umidade.

A principal vantagem da utilização da frequência respiratória como uma forma de determinação de situações de estresse térmico é devido ser um fácil parâmetro de aferição, sem a necessidade de equipamento adicional (BrownBrandl et al., 2005), onde o efeito da temperatura ambiente na frequência respiratória pode ser influenciado pela idade, sexo, genótipo, desempenho animal, nutrição, tempo de alimentação, escore corporal, sistema de criação e estratégias no uso de equipamentos de resfriamento. 
DANTAS, M.R.T. et al. Termorregulação de bovinos em ambiente tropical: uma abordagem com ênfase nas respostas fisiológicas. PUBVET, Londrina, V. 6, N. 7, Ed. 194, Art. 1306, 2012.

Estresse térmico provoca a ativação de mecanismos evaporativos de perda de calor envolvendo um aumento na freqüência respiratória (Kabuga, 1992; Gaughan et al., 1999, 2000; Hansen, 2004; Brown-Brandl et al., 2005; Beatty et al., 2006; Mader et al., 2006), e sudação (Hansen, 2004; Maia et al., 2005; Souza Jr, 2009; Silva e Maia, 2011). Valtorta et al. (1997), Eigenberg et al. (2000) e Brown-Brandl et al. (2005), verificaram em seus estudos que a frequência respiratória de bovinos mantidos à sombra foi menor do que bovinos mantidos sem sombreamento. Assim, bovinos expostos à radiação solar direta, ou seja, recebendo uma elevada carga de energia térmica, necessitam dissipar o excesso de calor contido em seus tecidos, sendo o aumento da frequência respiratória o primeiro sinal visível da situação de estresse térmico.

Estudos recentes tem sido realizados com o objetivo de avaliar a influência de diferentes ambientes nas respostas fisiológicas de bovinos. Em estudo realizado na Tailândia por Aengwanich et al. (2011), utilizando bovinos da raça Brahman, verificou-se reduções na frequência respiratória e sudação dos animais mantidos em sombreamento artificial e sombreamento de árvores quando comparados com bovinos expostos a radiação solar direta. Tais resultados foram de acordo com os valores observados por Eigenberg et al. (2009), na qual descobriram que o uso de estruturas para sombreamento artificial podem reduzir a incidência de radiação solar cerca de $30 \%$. Souza Jr (2009) estudando vacas Holandesas em ambiente semiárido verificou que a frequência respiratória apresenta uma relação positiva com a radiação solar quando os animais estão expostos ao sol, onde, nas horas mais quentes do dia, os animais estudados apresentaram um valor máximo de 105 resp. min $^{-1}$.

Outro fator importante que exerce influencia na frequência respiratória de bovinos é o horário do dia, na qual ocorrem variações nos níveis de radiação térmica, temperatura do ar e umidade relativa do ar. Segundo Oliveira et al. (2011), a frequência respiratória de bezerros mestiços de zebu $x$ bovinos apresentou valores médios mais elevados no período da tarde do que pela manhã. Cunha et al. (2007ab), também encontrou esta mesma relação. A 
elevação da carga térmica radiante e a temperatura do ar nestes horários fizeram com que os animais recebessem do ambiente maior quantidade de calor havendo a necessidade de dissipá-lo e, neste caso, o aumento da frequência respiratória indica elevação na perda de calor por evaporação respiratória.

\subsection{TEMPERATURA RETAL}

A temperatura retal é um indicador fisiológico utilizado pelo animal como a última resposta à tensão recebida do ambiente, ou seja, quando os mecanismos de dissipação térmica, principalmente a transferência de calor latente, já não são mais suficientes para manter a termorregulação (SCHARF et al., 2008). Segundo Kolb (1987), a temperatura retal média para bovinos acima de um ano é de $38,5 \pm 1,5^{\circ} \mathrm{C}$. Esta temperatura é mantida mediante regulação cuidadosa do equilíbrio entre a formação de calor e sua liberação do organismo. Segundo Dukes (1996), variações de 38,0 a $39,3^{\circ} \mathrm{C}$ na temperatura retal de bezerros de rebanhos leiteiros são consideradas normais. Os bovinos apresentam a capacidade de manter a temperatura corporal relativamente constante, porém, em condições de estresse térmico, dependendo da intensidade e da duração desse estresse, podem apresentar temperatura corporal elevada (BACCARI et al., 1995).

A medida da temperatura retal é usada freqüentemente como índice de adaptabilidade fisiológica aos ambientes quentes, pois seu aumento mostra que os mecanismos de liberação de calor tornaram-se insuficientes (MOTA, 1997). Silva (2000), entretanto, relatou que, em razão das diferenças na atividade metabólica dos diversos tecidos, a temperatura não é homogênea no corpo todo e varia de acordo com a região anatômica. As regiões superficiais apresentam temperatura mais variável e mais sujeita às influências do ambiente externo.

Segundo Aengwanich et al. (2011), em um estudo realizado com bovinos de corte, este não se surpreendeu que a temperatura retal dos bovinos sob 
DANTAS, M.R.T. et al. Termorregulação de bovinos em ambiente tropical: uma abordagem com ênfase nas respostas fisiológicas. PUBVET, Londrina, V. 6, N. 7, Ed. 194, Art. 1306, 2012.

sombra artificial, à sombra de árvores e sem sombra não foram significantemente diferentes entre si. Estes resultados também foram de acordo com os aferidos por Mitlohner et al. (2001), na qual observou que a temperatura retal não diferiu entre novilhas tratadas com sombreamento e sem sombreamento. Aengwanich et al. (2011) verificaram no dia 21 de seu experimento que a temperatura retal dos bovinos de corte sem sombreamento e os bovinos de corte mantidos abaixo de sombra de árvores forma maiores do que dos bovinos de corte mantidos à sombra artificial. Os resultados mostraram que, neste período, sombreamento artificial pode melhor proteger bovinos de corte do desconforto térmico ambiental do que o sombreamento pelas árvores. O acréscimo na temperatura retal dos bovinos sem sombreamento superou os bovinos mantidos sob sombreamento artificial.

No estudo de Souza Jr (2009) com vacas Holandesas em ambiente semiárido, observou-se que a temperatura retal de vacas Holandesas expostas ao sol e sob sombreamento artificial não teve diferença significativa quanto à temperatura retal. Isto já era um fato esperado devido os animais estarem protegidos da radiação solar direta, não sendo necessário elevar a temperatura retal devido não receber uma carga excessiva de energia térmica nessas condições. Já para os animais ao sol, devido os animais em estudo receber uma quantidade maior de calor proveniente da radiação direta, houve uma elevação na temperatura retal chegando a um valor máximo de $40,6^{\circ} \mathrm{C}$.

\subsection{TEMPERATURA SUPERFICIAL}

A temperatura de superfície corporal depende, principalmente, das condições ambientes de umidade e temperatura do ar e vento, e das condições fisiológicas, como vascularização e evaporação pelo suor. Assim, contribui para a manutenção da temperatura corporal mediante trocas de calor com o ambiente em temperaturas amenas. Os bovinos dissipam calor para o ambiente através da pele por radiação, condução e convecção, ou seja, perda de calor sensível (Cunningham, 1999). Sob condições de estresse pelo calor, 
DANTAS, M.R.T. et al. Termorregulação de bovinos em ambiente tropical: uma abordagem com ênfase nas respostas fisiológicas. PUBVET, Londrina, V. 6, N. 7, Ed. 194, Art. 1306, 2012.

as perdas sensíveis são diminuídas e a evaporação torna-se o principal processo de perda de calor.

A temperatura da superfície corporal dos animais influencia diretamente a troca de calor sensível. Sob altas temperaturas, esse mecanismo pode se transformar em uma via de ganho de calor (MAIA et al., 2005ab) e, nestas condições, o equilíbrio térmico pode ser afetado causando alterações diretas sobre a temperatura interna e a perda de calor por evaporação cutânea e respiratória. Souza Jr et al. (2008a) trabalhando com vacas Holandesas no Semi-Árido mostrou em seus resultados que é notório que à medida que o gradiente de temperatura, ou seja, que a temperatura da superfície do pelame se distancia da temperatura do ar há um aumento significativo na dissipação térmica convectiva e quando esse gradiente diminui, houve um decréscimo na perda de calor por convecção.

A temperatura da superfície do pelame está totalmente exposta às variações dos diferentes componentes meteorológicos que circundam o animal e, em vacas da raça Holandesa, a variação na temperatura do pelame é ainda mais acentuada devido esses animais possuírem uma pelagem composta por dois tipos de coloração, preta e branca, distribuídos de forma heterogênea por toda a superfície corporal (Souza Jr et al., 2008b). Sabe-se, que superfícies negras têm a capacidade de absorver uma maior quantidade de radiação que incidir sobre ela, mas no caso de superfícies claras, a maior parte da radiação que incidir sobre ela será refletida (Silva et al., 2003).

Silva et al. (2008) encontrou resultados que afirmam que a temperatura da superfície do pelame depende diretamente das condições do meio, ou seja, alterações bruscas das variáveis meteorológicas no local onde o animal se encontra, principalmente se o animal for exposto a céu aberto, haverá alterações da temperatura do pelame. Segundo Martello (2002), a temperatura da superfície corporal de vacas da raça Holandesa, alojadas em instalações climatizadas, pode variar de $31,6^{\circ} \mathrm{C}(6 \mathrm{~h})$ a $34,7^{\circ} \mathrm{C}(13 \mathrm{~h})$, sem indicar que o animal está sofrendo estresse pelo calor. Este trabalho teve o objetivo de 
DANTAS, M.R.T. et al. Termorregulação de bovinos em ambiente tropical: uma abordagem com ênfase nas respostas fisiológicas. PUBVET, Londrina, V. 6, N. 7, Ed. 194, Art. 1306, 2012.

estudar as respostas fisiológicas de bovinos cruzados F2 (1/2 Gir x 1/2 Holandês) submetidos ao estresse calórico.

O efeito da época do ano, horário do dia, cor e características do pelame sobre a temperatura da superfície corporal tem sido reportado em estudos recentes. Façanha et al. (2010) verificou em seu estudo que a temperatura na superfície do pelame branco foi aproximadamente $4^{\circ} \mathrm{C}$ inferior àquela observada no pelame preto dos animais avaliados (Figura 2). Silva et al. (2003), utilizando um espectro-radiômetro em comprimentos de onda entre 300 e $850 \mathrm{~nm}$, determinaram valores da absortância no pelame de animais holandeses no nível de 0,902 para o preto e 0,518 para o branco. A maior temperatura nas malhas negras decorre de sua maior absorção da radiação solar. Resultados similares foram observados por Pocay et al. (2001), porém os valores foram 44,59 e $38,18^{\circ} \mathrm{C}$ para temperatura da superfície do pelames preto e branco, respectivamente. Esta maior temperatura na superfície registrada por Pocay et al. (2001) reflete o horário em que a observação foi feita. No trabalho de Façanha et al. (2010) essas temperaturas foram registradas por volta das $9 \mathrm{~h}$, enquanto no trabalho de Pocay et al. (2001) foram anotadas por volta das $14 \mathrm{~h}$, ou seja no período do dia em que a intensidade de radiação solar na região é maior. Já Souza Jr et al. (2008b), estudando vacas Holandesas expostas a radiação solar direta em ambiente semiárido no mês de setembro, encontraram um valor máximo para temperatura de superfície do pelame branco e preto de $40,6^{\circ} \mathrm{C}$ e $42,9^{\circ} \mathrm{C}$ às $13 \mathrm{~h}$, respectivamente. A diferença média das temperaturas do pelame branco e preto de vacas Holandesas é de aproximadamente $2,3^{\circ} \mathrm{C}$ (Souza Jr et al., 2008b).

Façanha et al. (2010) estudaram a variação anual da temperatura da superfície e características morfológicas do pelame de vacas leiteiras em ambiente semiárido, onde, no mês de junho a temperatura da superfície do pelame (Figura 2) foi $1,5^{\circ} \mathrm{C}$ mais baixa no pelame branco e $2,5^{\circ} \mathrm{C}$ no preto, diferenças menores que as observadas nos demais meses. Esses resultados comprovam que, no período em que os pelames eram densos, eles 
DANTAS, M.R.T. et al. Termorregulação de bovinos em ambiente tropical: uma abordagem com ênfase nas respostas fisiológicas. PUBVET, Londrina, V. 6, N. 7, Ed. 194, Art. 1306, 2012.

apresentavam menor temperatura na sua superfície. O mesmo foi observado por Gebremedhin et al. (1997) em pelame com espessura de até $6 \mathrm{~mm}$. A temperatura da superfície do pelame preto foi em média $4^{\circ} \mathrm{C}$ maior que a do pelame branco (Figura 2) e ambas acompanharam a temperatura radiante média. Este resultado está de acordo com Pocay et al. (2001) e Hillman et al. (2001). No entanto, o estudo de Façanha et al. (2010) comprova que essa diferença de temperatura ocorre no mesmo animal, ou seja, a temperatura na malha preta é $4,0^{\circ} \mathrm{C}$ mais elevada que na branca no mesmo indivíduo, uma vez que a cor branca reflete grande parte da radiação incidente, ao passo que a cor negra das malhas absorve a maior parte da radiação de ondas curtas que se transforma em energia calorífica, elevando a temperatura da superfície.

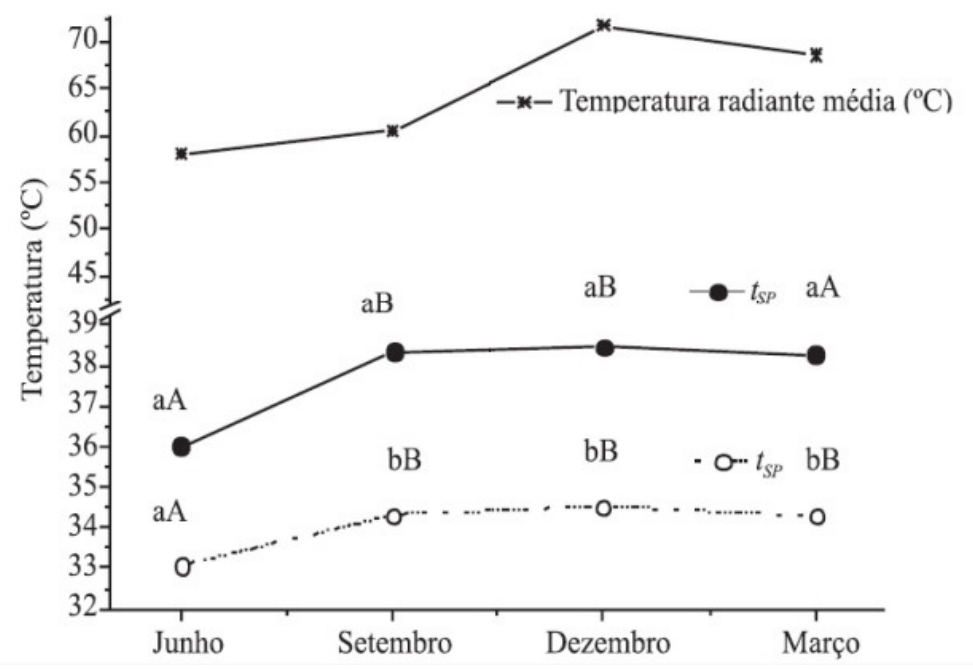

Figura 2 Efeito da interação do mês de coletas com temperatura dos pelames branco e preto de vacas leiteiras. Adaptado de Façanha et al. (2010).

\section{CONSIDERAÇÕES FINAIS}

Os efeitos do ambiente térmico sobre as respostas fisiológicas de bovinos leiteiros tem sido bastante estudados como uma forma de caracterizar situações de estresse térmico causado por tensões exercidas pelo ambiente o 
DANTAS, M.R.T. et al. Termorregulação de bovinos em ambiente tropical: uma abordagem com ênfase nas respostas fisiológicas. PUBVET, Londrina, V. 6, N. 7, Ed. 194, Art. 1306, 2012.

qual os animais estão inseridos. A temperatura superficial, por estar diretamente exposta ao ambiente, apresenta variações consideráveis, as quais acompanham as variações dos componentes meteorológicos do ambiente em que estão inseridos. Já a temperatura retal é uma resposta tardia ao estresse térmico, com pouca ou nenhuma variação. Ao contrário da temperatura retal, a frequência respiratória é o primeiro sinal visível que evidencia uma situação de estresse calórico. Assim, para evitar situações térmicas que impeçam os animais de demonstrarem todo o seu potencial produtivo, além de evitar danos na saúde e reprodução, deve-se fornecer um microclima caracterizado por baixas temperaturas e sem a exposição à radiação solar direta.

\section{REFERÊNCIAS}

Arkin H. et al. 1991. Heat transfer properties of dry an wet furs of dairy cows. Transactions of the ASAE, v. 34, p. 2550-2558.

Aengwanich W. et al. 2011. Effects of shade on physiological changes, oxidative stress, and total antioxidant power in Thai Brahman cattle. Int J Biometeorol (2011) 55:741-748.

Baccari Jr.F. et al. 1995. Hipertermia, taquipnéia e taquicardia em vacas holandesas malhadas de vermelho sob stress. In: I Congresso Brasileiro De Biometeorologia, Jaboticabal. Anais..., Jaboticabal: SBBIO, p. 15-26.

Baeta F.C. \& Souza C.F. 1997. Ambiência em edificações rurais: conforto animal. Viçosa: UFV, 1997. p. 25.

Baker M.A. 1989. Effect of dehydration and rehydration on thermoregulatory sweating goats. J. Physiol. Lond., v. 417, p. 421-435.

Beatty D.T., et al. 2006. Physiological responses of Bos taurus and Bos indicus cattle to prolonged, continuous heat and humidity. J Anim Sci 84:972-985

Bianca W. 1965. Reviews of the progress of dairy science. Section A. Physiology. Cattle in a hot environmental. J. Dairy Res., v.32, p.291-345.

Borut A. et al. 1979. Heat balance of resting and walking goats: comparison of climatic chamber and exposure in the desert. Physiology Zoology, v.52, p.105-112.

Brown-Brandl T.M. et al. 2005. Dynamic response indicators of heat stress in shaded and nonshaded feedlot cattle, Part 1: analyses of indicators. Biosys Eng 90:451-462.

Carvalho F.A. et al. 1995. Breed effects thermoregulation and epithelial morphology in imported and native cattle subjected to heat stress. J. Anim. Sci., v.73, p.3570-3573. 
Cunha D.N.F.V. et al. 2007a. Desempenho, variáveis fisiológicas e comportamento de bezerros mantidos em diferentes instalações: época chuvosa. Rev. Bras. Zootec. v.36, n.4, p.11401146.

Cunha D.N.F.V. et al. 2007b. Desempenho, variáveis fisiológicas e comportamento de bezerros mantidos em diferentes instalações: época seca. Rev. Bras. Zootec. v.36, n.4, p.847-854.

Cunningham, J.G. 1999. Tratado de fisiologia veterinária. 2.ed. Rio de Janeiro: Guanabara Koogan. 454p.

Curtis S.E. 1983. Environmental management in animal agriculture. AMES: The Iowa State University Press, 409 p.

Dukes H.H. 1996. Fisiologia dos animais domésticos. 11ed. Rio de Janeiro: Guanabara Koogan, $856 \mathrm{p}$.

Eigenberg R.A. et al. 2000. Development of a new respiration rate monitor for cattle. Trans ASAE 43:723-728.

Eigenberg R.A. et al. 2009. Shade material evaluation using a cattle response model and meteorological instrumentation. Int J Biometeorol 53:501-507.

Ehrlemark A.G. \& Sallvik K.G.A. 1996. model of heat and moisture dissipation from cattle based on thermal properties. Transactions of the ASAE., v. 39, p. 187-194.

Esmay M.L. 1969. Principles of animal environment. Westport: AVI.

Dmi'el R. \& Robertshaw D. 1983. The control of panting and sweating in the black Bedoin goat: A comparison of two modes of imposing a heat load. Physiol. Zool., 56: 404-411.

Ferreira F. et al. 2006. Parâmetros fisiológicos de bovinos cruzados submetidos ao estresse calórico. Arquivo Brasileiro de Medicina Veterinária e Zootecnia, v.58, n.5, p.732-738.

Finch V.A. et al. 1982. Sweating response in cattle and its relation to rectal temperature, tolerance of sun and metabolic rate. Journal of Agricultural Science, v. 99, p. 479-487.

Finch V.A. et al. 1984. Coat color in cattle: effect of thermal balance, behaviour and growth and relationship with coat type. Journal of Agricultural Science, Cambridge, v. 102 p. 141-147.

Finch V.A. 1985. Comparison of non-evaporative heat transfer in different cattle breeds. Australian Journal of Agricultural Research, v. 36, p. 497-508.

Garcia-Ispierto I. et al. 2005. Relationship between heat stress during the peri-implantation period and early fetal loss in dairy cattle. Theriogenology, Stoneham, v. 65, p. 799-807.

Gaughan J.B. et al. 1999. Heat tolerance of Boran and Tuli crossbred steers. J Anim Sci 77:2398-2405.

Gaughan J.B. et al. 2000. Valtorta. et al. 1997. Evaluation of different shades to improve dairy cattle well-being in Argentina. Int J Biometeorol 41:65-67.

Gayão, A.L.B.A. 1992. Efeito do estresse térmico sobre a taxa metabólica e o desempenho produtivo de cabritas Saanen em crescimento. 68f. Dissertação (Mestrado em Zootecnia) Faculdade de Medicina Veterinária e Zootecnia, Universidade Estadual Paulista, Botucatu-SP. 
Gebremedhin K.G. et al. 1981. Predictions and measurements of heat production and food and water requirements of Holstein calves in different environments. Transactions of the ASAE, v. 3 p. 715-720.

Gebremedhin K.G. et al. 1997. Modeling temperature profile and heat flux through irradiated fur layer. Transactions of the ASAE, v.40, n.5, p.1441-1447.

Gebremedhin K.G. \& Wu B. 2001. A model of evaporative cooling of wet skin surface and fur layer. Journal of Thermal Biology, v. 26, p. 537-545, 2001.

Hansen P.J. 2004. Physiological and cellular adaptations of zebu cattle to thermal stress. Anim Reprod Sci 82-83:349-360 Janzekovic. et al. 2006. Techniques of measuring heart rate in cattle. Tehnicki Vjesnik 13:31-37.

Hillman P.E. et al. 2001. Impact of hair colour on thermoregulation of dairy cows to direct sunlight. In: Annual International Meeting Of The Asae, 94., Sacramento. Proceedings... Sacramento, 2001. (Paper, 014301). p.14.20.

Igono M.G. et al. 1985. Spray cooling effects on milk production, milk and rectal temperatures of cows during a moderate temperature summer season. J. Dairy Sci., v.68, p.979-985.

Ingraham R.H. et al. 1979. Seasonal effects on shade and nonshade cows as measure by rectal temperature, adrenal cortex hormones, thyroid hormone and milk production. Am. J. Vet. Res., v.40, p.1792-1797, 1979.

Jessen C. \& Pongratz H. 1979. Air humidity and carotid rate function in thermoregulation of the goat. Journal Physiology, v. 292. p. 469-479.

Johnson H. D. 1987. Bioclimatology and adaptation of livestock. Amsterdam: Elsevier. 279 p. 56.

Jordan E. R. 2003. Effects of heat stress on reproduction. Journal of Dairy Science, Champaign, v. 86, p. $104-114$.

Kabuga J.D. 1992. The influence of thermal conditions on rectal temperature, respiration rate and pulse rate of lactating Holstein-Friesian cows in the humid tropics. Int J Biometeorol 36:146-150.

Kolb E. Fisiologia Veterinária. 4. ed., Rio de Janeiro: Guanabara Koogan, 1987. 612p.

Littell R.C. et al. 1991. SAS ${ }^{\circledR}$ System for Linear Models, 3.ed. Cary, NC: SAS Institute Inc.. 329p.

Mader T.L. et al. 2006. Environmental factors influencing heat stress in feedlot cattle. J Anim Sci 84:712-719.

Maia A.S.C. et al. 2005a. Sensible and latent heat loss from body surface of Holstein cows in a tropical environment. International Journal of Biometeorology, v. 50, p. 17-22.

Maia A.S.C. et al. 2005b. Respiratory heat loss of Holstein cows in a tropical environment. International Journal of Biometeorology, v. 49, n. 5, p. 332-336.

Marai I.F.M. et al. 1999. Productive, physiological and biochemical changes in imported an locally born Holstein lactating cows under hot summer conditions of Egypt. Trop. Anim. Health Prod., v.31, p.233-243. 
Martello L.S. 2002. Diferentes recursos de climatização e sua influência na produção de leite, na termorregulação dos animais e no investimento das instalações. 2002. 67f. Dissertação (Mestrado) - Faculdade de Zootecnia e Engenharia de Alimentos, Universidade de São Paulo, Pirassununga, SP.

Martello L.S. et al. 2004. Respostas fisiológicas e produtivas de vacas holandesas em lactação submetidas a diferentes ambientes. Revista Brasileira de Zootecnia, v. 33, n. 1, p. 181-191.

McLean J.A. 1963a. Measurement of cutaneous moisture vaporization from cattle by ventilated capsules. Journal of Physiology, v. 167, p. 417-426.

McLean J.A. 1963b. The partition of insensible losses of body weight in heat from cattle under various climatic conditions. Journal of Physiology, v. 167, p. 427-447.

Mitlohner F.M. et al. 2001. Shade and water misting effects on behavior, physiology, performance, and carcass traits of heatstressed feedlot cattle. J Anim Sci 79:2327-2335.

Morberg G.P. 1976. Effects of environment and management stress on reproduction in dairy cow. Journal of Dairy Science, Champaign, v. 59, p. 1618-1624.

Mota L.S.L.S. 1997. Adaptação e interação genótipo-ambiente em vacas leiteiras. 69p. Tese (Doutorado em Medicina Veterinária) - Faculdade de Medicina Veterinária de Ribeirão Preto, Universidade de São Paulo, Ribeirão Preto.

Muller C.J. \& Botha J.A. 1993. Effect of summer climatic conditions on different heat tolerance indicators in primiparous Friesian and Jersey cows. S. Afr. J. Anim. Sci., v.23, p.98-103.

Muller C.J. et al. 1994a Effect of shade on various parameters of Friesian cow in a Mediterranean climate in South Africa. 2: Physiological responses. S. Afr. J. Anim. Sci., v.24, p.56-60.

Muller C.J.C. et al. 1994b. Production, physiological and behavioral responses of lactating Friesian cows to a shade structure in a temperate climate. in: bucklin, r.a. (ed.). international dairy housing conference, 3.,1994b, St. Joseph, MI. Proceeding... St. Joseph, MI: American Society of Agricultural Engineers. p.597-588.

Nääs I.A. et al. 2001. Avaliação térmica de telhas de composição de celulose e betume, pintadas de branco, em modelos de aviários com escala reduzida. Engenharia Agrícola, Jaboticabal, v.21, n.2, p.121-6.

Okantah S.A. et al. 1992. The effect of diurnal changes in ambient temperature on heat tolerance in some cattle breeds and crossbreeds in a tropical environment. Bull. Anim. Health Prod. Afr., v.41, p.33-38.

Oliveira F.C.S. et al. 2011. Respostas fisiológicas de bezerros mestiços em uma região de baixa latitude. In: 48. Reunião Anual da Sociedade Brasileira De Zootecnia, 48. Belém/PA. Anais..., Belém: SBZ. 2011. p. 238-240.

Pires M.F.A. et al. 1998a. Estresse calórico em bovinos de leite. In: SIMPÓSIO DE PRODUÇÃO E NUTRIÇÃO DE GADO LEITEIRO, 1998a, Belo Horizonte. Anais... Belo Horizonte. p.17-30.

Pires M.F.A. et al. 1998b. Efeito das estações (inverno e verão) na temperatura retal e frequência respiratória de vacas Holandesas confinadas em free stall. Arq. Bras. Med. Vet. Zootec., v.50, p.747-752. 
Pocay P.L.B. et al. 2001. Respostas fisiológicas de vacas Holandesas predominantemente brancas e predominantemente negras sob radiação solar direta. Ars Veterinaria, v.17, n.2, p.155-161.

Santos E.L. 2003. Efeito do estresse calórico em caprinos. 66f. Dissertação (Mestrado em Zootecnia) - Faculdade de Zootecnia e Engenharia de Alimentos, Universidade de São Paulo, Pirassununga-SP.

Scharf B. et al. 2008. Regional differences in sweat rate response of steers to short-term heat stress. Int. J. Biometeorol. v. 52, p. 725-732.

Schmidt-Nielsen K. 2002. Fisiologia animal: adaptação e ambiente, 5th ed. Cambridge: Cambridge Univ. Press, 611p.

Silva R.G. 1993. Manual de procedimentos em análise por quadrados mínimos. Jaboticabal: FUNEP, 169p.

Silva R.G. 2000a. Um modelo para a determinação do equilíbrio térmico de bovinos em ambientes tropicais. Revista Brasileira de Zootecnia, v.29, n. 4 p.1244-1252.

Silva R.G. 2000b. Introdução à bioclimatologia animal. São Paulo: Nobel/FAPESP, 286p..

Silva R.G. et al. 2002. Respiratory heat loss in the sheep: a comprehensive model. International Journal of Biometeorology, v.4, p.136-140.

Silva R.G. \& Starling J.M.C. 2003. Evaporação cutânea e respiratória em ovinos sob altas temperaturas ambientes. Revista Brasileira de Zootecnia, v.32, n.6, p.1956-1961, (supl. 2).

Silva R.G. 2008. Biofísica ambiental: os animais e seu ambiente. São Paulo: FUNEP. 386p.

Silva R.G. \& Maia A.S.C. 2011. Evaporative cooling and cutaneous surface temperature of Holstein cows in tropical conditions. Revista Brasileira de Zootecnia, vol.40, n.5, pp. 11431147. ISSN 1806-9290.

Souza Jr. J.B.F. et al. 2008a. Temperatura da superfície corporal e fluxo de calor convectivo em vacas Holandesas expostas à radiação solar direta no Semi-Árido. In: In: CONGRESSO NORDESTINO DE PRODUÇÃO ANIMAL, 5., 2008a, Aracajú/SE. Anais..., Aracajú: SNPA. p. 232-234.

Souza Jr. J.B.F. et al. 2008b. Variação diária da temperatura da superfície do pelame preto e branco de vacas Holandesas no Semi-Árido. In: CONGRESSO NORDESTINO DE PRODUÇÃO ANIMAL, 5., Aracajú/SE. Anais..., Aracajú: SNPA. 2008. p. 238-240.

Souza Jr. J.B.F. 2009. Estudo da perda de calor por evaporação na superfície corporal em vacas holandesas manejadas no Semi-árido. 49f. Monografia (Graduação em Zootecnia) Universidade Federal Rural do Semi-Árido, Mossoró-RN.

Stevens D.G.A. 1981. Model of respiratory vapor loss in Holstein dairy cattle. Transactions of the ASAE, v.24 p.151-158.

Stöber M. 1993. Identificação, anamnese, regras básicas da técnica de exame clínico geral. In: Dirksen G.; Gründer H.D.; Stöber M. Exame clínico dos bovinos. 3.ed. Rio de Janeiro: Guanabara Koogan. cap.2, p.44-80. 
Terra R.L. 1993. História, exame físico e registro dos ruminantes. In: Smith B.P. Tratado de medicina interna dos grandes animais. São Paulo: Manole. v.1, cap.1, p.3-15.

Taneja G.C. 1958. Cutaneous evaporative losses in calves and its relationship with respiratory evaporative loss and skin and rectal temperatures. Journal of Agricultural Science, v. 50, Part.1, p. 73-85.

Taneja G.C. 1959. Cutaneous evaporative loss measured from limited areas and its relationship with skin, rectal, and air temperatures. Journal of Agricultural Science, v. 52, p. 50-61.

Wolff L.K. \& Monty D.E. 1974. Physiologic responses to intense summer heat stress and its effect on the estrous cycle of non-lactating and lactating Holstein-Friesian cows in Arizona. Am. J. Vet. Res., v.35, p.187-192.

Yousef M.K. 1987. Principles of bioclimatology and adaptation. Word Animal Science, v. 5, p.17-29. 